\title{
A broadband NMR spectrometer for field stabilization in the range 4-23 kG
}

\author{
Roger M. Hawk, Robert R. Sharp, and John W. Tolan \\ Depatment of Chemistry, University of Michigan, Ann Arbor, Michigan 48104 \\ (Received 13 July 1973; and in final form, 15 August 1973)
}

\begin{abstract}
A broadband "time-shared" NMR spectrometer has been constructed for the purpose of magnetic field stabilization. An error signal for field correction is derived from the ${ }^{7} \mathrm{Li}$ or ${ }^{1} \mathrm{H}$ resonance of a saturated $\mathrm{LiCl}$ solution in formamide. The unit has been tested only under low resolution conditions using a $24 \mathrm{~cm}$ magnet, but operates well in pulsed and wide-line experiments at fields in the range 4-21 kG. Integrated circuitry is used throughout, and the design is relatively simple and inexpensive to construct.
\end{abstract}

\section{INTRODUCTION}

High resolution NMR experiments are normally carried out at the highest attainable field strength in order to maximize chemical shifts and signal-to-noise ratios. $\mathrm{Re}$ laxation time measurements, on the other hand, are often field-dependent, and an ability to perform measurements throughout a range of stabilized fields is useful in such cases to obtain a complete analysis of the data. Many examples of field-dependent relaxation may be cited. In our own research, which involves relaxation studies of spin- $\frac{1}{2}$ metal nuclei, variable field operation has been an invaluable aid in distinguishing the field-dependent $T_{1}$ mechanisms (scalar coupling and chemical shift anisotropy) from those that are field-independent, such as dipolar and spin-rotation interactions.

This paper describes the construction of a broad-band external nmr field lock that has been used for pulsed measurements at fields ranging from 4 to $21 \mathrm{kG}$. The device is based on the "time-shared" technique of Baker et $a l .{ }^{1}$ and is designed for detection of nuclear resonances in the range $16-40 \mathrm{MHz}$. Time-shared modulation functions by switching on the rf transmitter periodically and simultaneously inactivating the receiver. If rf switching is complete, only the free-induction decay (FID) is ob- served between pulses. The FID is then phase-detected in the $\mathrm{rf}$ and audio regions (audio phase detection is referred to the frequency of $\mathrm{rf}$ pulses), after which the filtered signal consists of side-band spectra similar to those observed in continuous wave nmr.

The field lock, which is similar in some respects the design of Ellett et al., ${ }^{2}$ is presently operated in conjunction with a Bruker B-KR-322s variable frequency (4-60 MHz) pulsed spectrometer. This system permits long-term data accumulation on many magnetic nuclei throughout a substantial range of magnetic field strengths.

\section{CIRCUIT DESCRIPTION}

Figure 1 contains a block diagram of the field locking device. Radio frequencies are derived from an external synthesizer (when this is available) or from interchangeable fixed frequency crystal oscillators. ${ }^{3}$ The rf input (approximately $1 \mathrm{~V} \mathrm{p}$-p) is split immediately into parallel channels, one of which leads to the transmitter, the other to the reference input of the rf phase detector. These channels are buffered by an identical pair of broad-band rf amplifiers (Fig. 2). The level in the transmitter channel is further amplified to about $4 \mathrm{~V}$ p-p and gated by a diode switch (1) shown in Fig. 3. Individual components of
Fig. 1. Block Diagram of the nmr spectrometer.

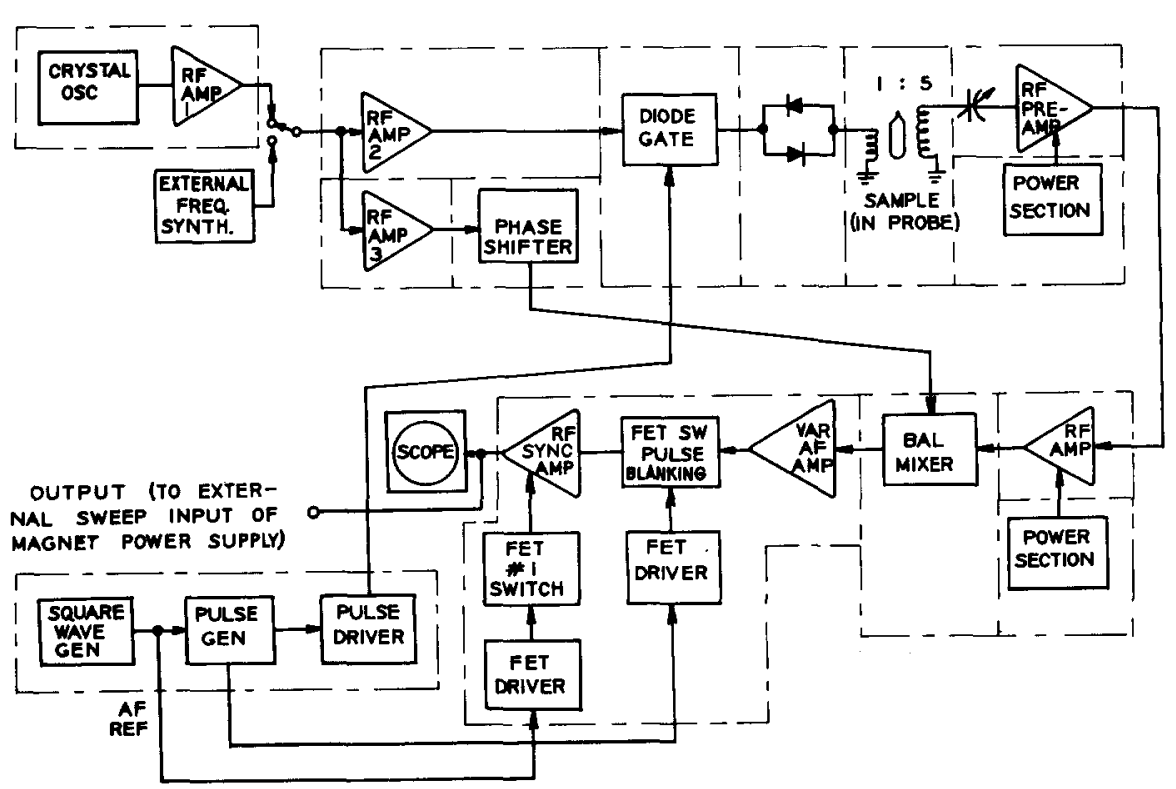




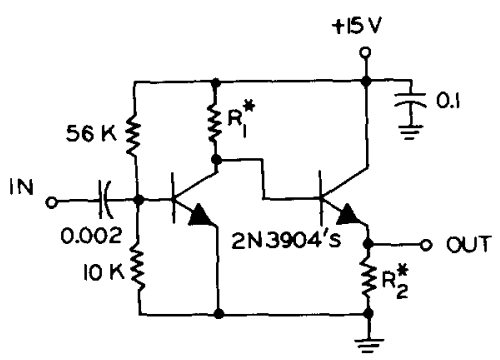

Fig. 2. Broad-band $r$ buffer amplifier. $R_{1}=270$ $\Omega, R_{2}=330 \Omega$ in amplifier $1 ; R_{1}=330 \Omega, R_{2}=330 \Omega$ in amplifier $2 ; R_{1}=330 \Omega$, $R_{2}=220 \Omega$ in amplifier 3 .

this switch are isolated in different compartments of an enclosure milled from solid aluminum. The switch requires $\pm 2 \mathrm{~mA}$ in its on/off states and provides $60 \mathrm{~dB}$ of $\mathrm{rf}$ isolation. Isolation during the off period is further augmented by a pair of crossed diodes (1N4448) placed in series with the transmitter coil.

Modifications of the Bruker probe assembly required for installation of the reference sample are shown in Fig. 4. A compartmented aluminum box is fastened to the rear of the probe head ${ }^{4}$ and contains the tuning capacitor with two stages of rf preamplification. The reference solution itself sits in a separate machined brass box immediately behind the sample insert inside the Bruker probe head. Receiver and transmitter coils are wound directly and concentrically on a $2.5 \mathrm{~cm}$ length of glass tubing, which contains the reference solution and is sealed at both ends. Two sets of coils permit tuning over the $16-40 \mathrm{MHz}$ range. The receiver coil consists of 40 turns of No. 30 enameled copper wire for the $16-24 \mathrm{MHz}$ range and 23 turns of No. 26 wire for the $24-40 \mathrm{MHz}$ range. Transmitter coils of 8 turns and 5 turns, respectively, are wound concentrically on the receiver coils near the grounded end and secured with "Q-dope."

A number of reference solutions were examined before one was found which exhibited satisfactory relaxation characteristics for both ${ }^{7} \mathrm{Li}$ and ${ }^{1} \mathrm{H}$ resonances. A saturated aqueous solution of $\mathrm{LiCl}$ doped with paramagnetic ions shortens the proton $T_{1}$ to millisecond values, but scarcely affects the ${ }^{7} \mathrm{Li}$ resonance. ${ }^{5}$ Comparable relaxation times can be obtained from a saturated solution of $\mathrm{LiCl}$ in formamide. The solution is prepared at $70^{\circ} \mathrm{C}$ by vigorous stirring and gels upon cooling to room temperature. Relaxation times of approximately $30 \mathrm{msec}$ are obtained for both ${ }^{7} \mathrm{Li}$ and ${ }^{1} \mathrm{H}$.

The resonance signal is preamplified at the probe (but outside the magnetic field) by a high gain ( $40 \mathrm{~dB}) \mathrm{Sig}$ netics NE592K broad-band video amplifier preceded by an emitter-follower buffer stage. A second buffered video

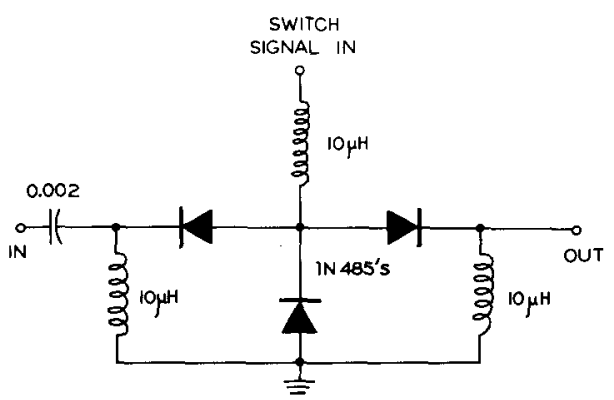

FIG. 3. Diode switch. amplifier is located in a box mounted on the main-frame and provides $40 \mathrm{~dB}$ further broad-band amplification.

A stable locking condition requires that the resonance signal be phase-detected for the dispersion mode. Both the side-bands and center-band appear in dispersion mode when the rf reference is $90^{\circ}$ out of phase with respect to the signal and the audio reference is $90^{\circ}$ out of phase with respect to the rf pulse sequence. ${ }^{6}$ A fixed rf phase angle is quite difficult to achieve in a variable frequency system, and for this reason an adjustable delay line ${ }^{7}$ is inserted in the reference channel. The rf phase is easily adjusted by monitoring the free induction decay, which is nulled, at resonance, for $90^{\circ}$ and $270^{\circ}$ phase angles.

The amplified rf signal is phase-detected by a HewlettPackard model 10543B double-balanced mixer located on a board with the audio circuitry in an aluminum box mounted on the main-frame. Input levels to the 10534B from the rf reference and from the output of the second video amplifier should be comparable in amplitude but limited to $0.5 \mathrm{~V}$ p-p for best operation and burnout protection. The audio output of the mixer is amplified by a Motorola MC 1439L op-amp, the output of which is switched off at twice the rf pulse repetition rate by a 2N5459 FET. Variable gain and offset controls for the op-amp are available at the front panel. The phase-detected signal, blanked during rf pulses, is coupled to a second MC 1439L operational amplifier which serves as an audio synchronous detector. This op-amp multiplies the incoming signal by \pm 1 depending on the logic state of a reference square wave which drives FET F-2. Its output, when filtered, provides synchronous amplification at the square wave frequency. A $90^{\circ}$ phase shift is introduced into the audio reference by logic circuitry described below. The output of the audio synchronous amplifier is filtered as required and provides a control signal for the sweep input of the magnet power supply. Figure 5 contains a schematic of the receiver circuitry.

Timing of the transmitter and receiver gate pulses and of the reference square wave is derived from logic circuits shown in Fig. 6. The clock is an IC function generator (Signetics NE566), the square wave output of which can be varied in frequency from 1 to $10 \mathrm{kHz}$ by front panel controls. Figure 6 compares the various gate and reference waveforms referred to above. The receiver is gated at twice the frequency of the rf pulses so that transients will occur symmetrically in both cycles, \pm 1 , of synchronous amplification. The receiver gate also leads and Jags the transmitter gate by a few microseconds to eliminate transients caused by recovery of the preamplifiers. Gate pulses to the transmitter are variable in width from $50 \mu \mathrm{sec}$ to $1 \mathrm{msec}$. This range is adequate to achieve $90^{\circ}$ pulse widths for both ${ }^{1} \mathrm{H}$ and ${ }^{7} \mathrm{Li}$ with a $4 \mathrm{~V}$ p-p rf input to the transmitter coil.

\section{CONSTRUCTION AND PERFORMANCE}

Each of the circuits involving rf was constructed on Vectorboard and isolated in a compartmented aluminum box, as noted above. Three aluminum boxes, the logic 
FIG. 4. Probe assembly containing reference sample preamplifier.

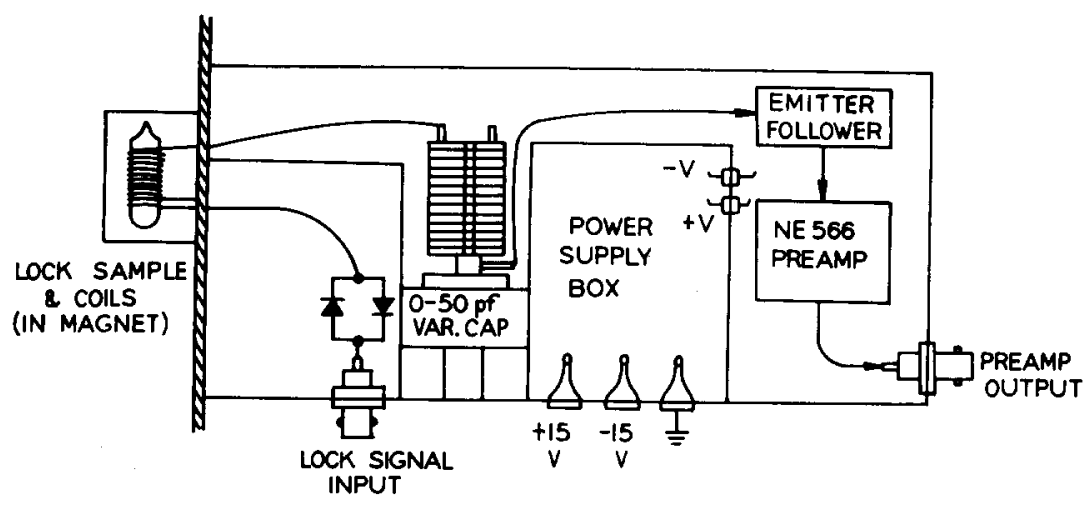

circuitry and a $\pm 15 \mathrm{~V}, 5 \mathrm{~A}$ power supply ${ }^{8}$ are mounted on a $48 \mathrm{~cm}$ panel. Chokes and bypass capacitors were used liberally to isolate dc lines to the various units.

The signal-to-noise ratio of the FID at $22 \mathrm{MHz}$ is approximately $40: 1$ for ${ }^{1} \mathrm{H}$ and $5: 1$ for ${ }^{7} \mathrm{Li}$ with a $100 \mathrm{kHz}$ bandwidth. Stable operation of the closed loop feedback system requires that the transfer functions of the regulator, the magnet, and the magnet power supply satisfy the Nyquist stability criterion; namely, that the phase lag of the open loop frequency response of the system at the frequency producing a log-modulus of $0 \mathrm{~dB}$ must be less than $180^{\circ}$. For acceptable operation in practise, the phase margin and gain margin should be considerably more conservative than those that define the Nyquist criterion. ${ }^{9}$ The analysis of loop gain and phase compensation normally involves an experimental determination of the transfer function of the specific system being regulated. This measurement is conveniently made by monitoring the frequency response of the control resonance to an imposed sinusoidal control signal of frequency $\omega$. The system's stability can then be analyzed from a Bode plot or a Nyquist diagram of the frequency dependence of the transfer function. Interested readers are referred to standard texts on feedback control theory.$^{9-11}$ The system described here operates satisfactorily if the regulator output is returned directly to the external sweep input of the magnet power supply (Varian model V-2501) providing the gain is kept below the threshold for oscillation. The lock has not been tested for stability under high resolution conditions, and at present the long-term field stability $( \pm 25 \mathrm{~Hz})$ is severely limited by the very poor field homogeneity ( 5 ppm over $1 \mathrm{~cm}^{3}$ ) of our $24 \mathrm{~cm}$ magnet.

A number of observations have been made on the ${ }^{119} \mathrm{Sn}$ resonance while locking on ${ }^{7} \mathrm{Li}$. The gyromagnetic ratios of these nuclei are very close together $(1587$ and $1655 \mathrm{~Hz} / \mathrm{G}$, respectively), and special precautions were required to ensure rf isolation inside the probe head. Although the lock sample was completely sealed inside a brass compartment, appreciable cross modulation between the coils was noted. Cross modulation was eliminated by shielding the Bruker

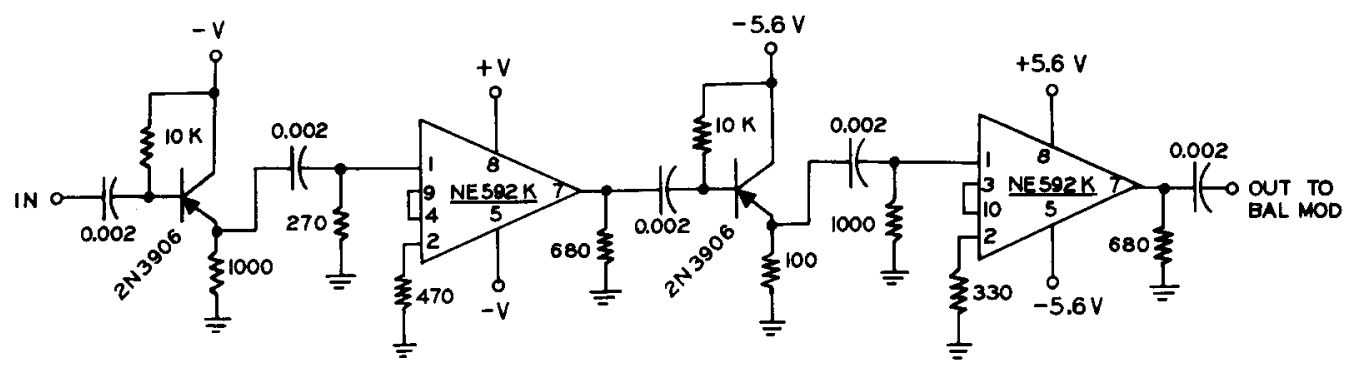

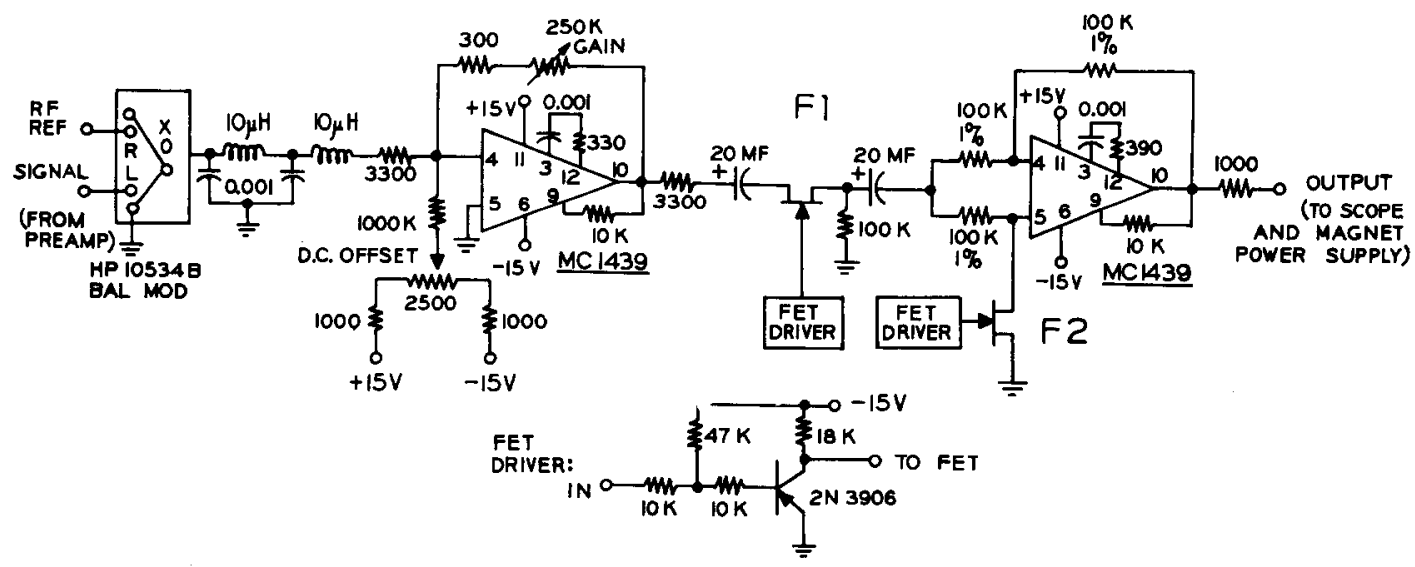

FIG. 5. Receiver circuit; F-1 and F-2 are 2N5459's. 


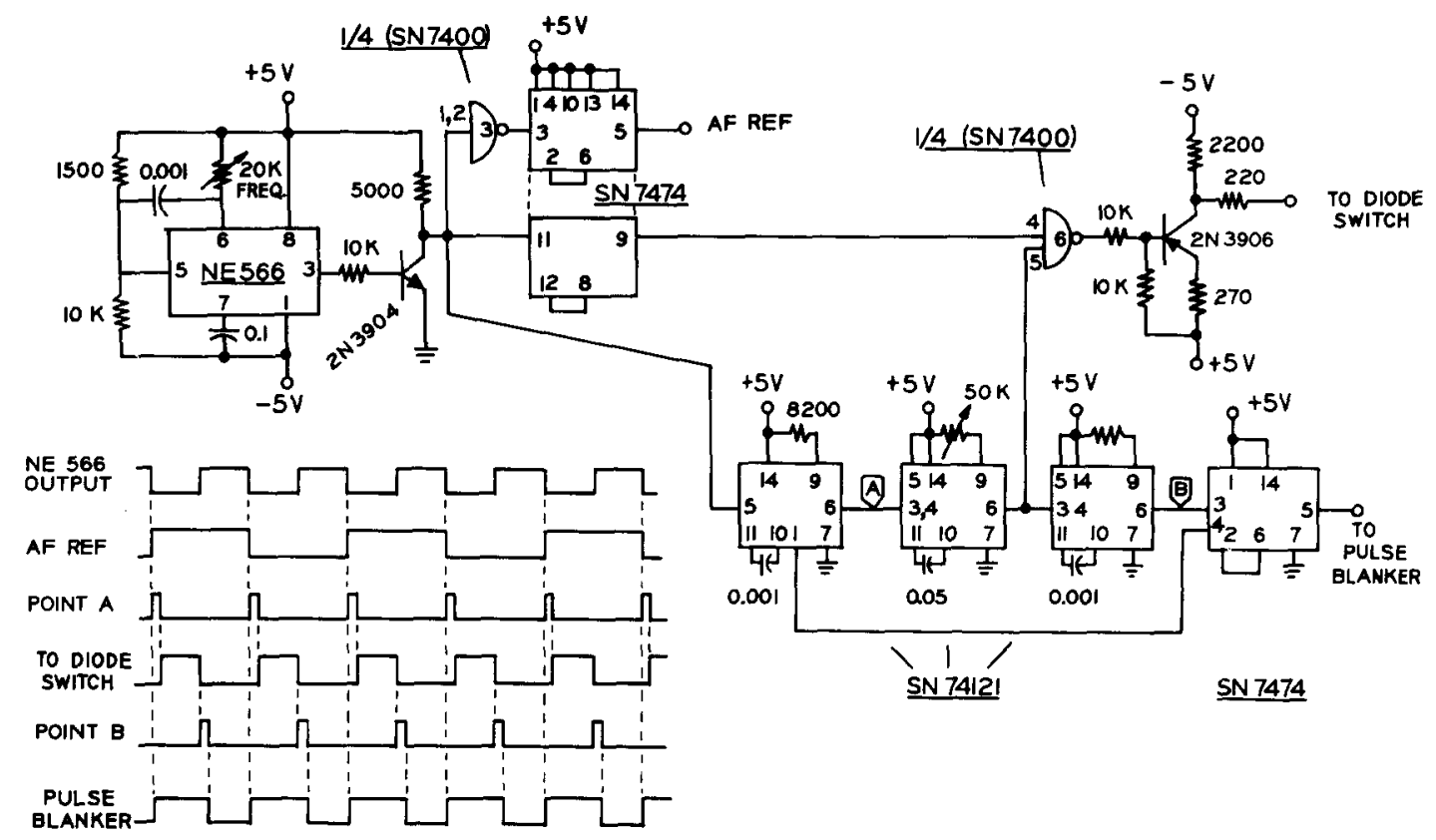

FIG. 6. Logic circuits from which gate and reference pulses are derived.

insert with a single layer of silver foil and the lock compartment with a layer of copper foil. This shielding would probably not be necessary, even at very similar frequencies, if the lock compartment were machined from high quality copper.

\section{ACKNOWLEDGMENTS}

The authors gratefully acknowledge financial support from the National Science Foundation and from the donors of the Petroleum Research Fund, administered by the American Chemical Society. Partial support of this work was also provided by the Rackham Foundation and the Memorial Phoenix Project of the University of Michigan.
${ }^{1}$ E. B. Baker, L. W. Burd, and G. M. Root, Rev. Sci. Instrum. 36, 1495 (1965)

${ }^{2}$ J. D. Ellett et al., Adv. Magn. Reson. 5, 117 (1971).

${ }^{3}$ International Crystal Manufacturing Co., 18 North Lee, Oklahoma City, Okla.

${ }^{4}$ Mounting this box requires that part of a support for the Dewared air heater of the Bruker temperature controller be removed.

${ }^{5}$ E. A. Nikiforov and A. A. Popel, J. Struct. Chem. 11, 1043 (1970).

${ }^{6} \mathrm{O}$. Haworth and R. E. Richards, Prog. Nucl. Magn. Reson. Spectrosc. 1, 1 (1966).

${ }^{7}$ Continuously variable delay line, ESC Corp., Palisades Park, N.J.; No. 71-96, total delay $=0.4 \mu$ sec.

${ }^{8}$ Electrostatics, Inc., San Diego, Cal.; model 50-1515.

${ }^{9} \mathrm{P}$. Atkinson, Feedback Control Theory for Engineers (Plenum, New York, 1968)

${ }^{10} \mathrm{~J}$. J. Di Stefano III, A. R. Stubberud, and I. J. Williams, Feedback and Control Systems, Schaum's Outline Series (McGraw-Hill, New York, 1967).

11 J. B. Cruz, Jr., Feedback Systems (McGraw-Hill, New York, 1972). 